\title{
The RMS survey
}

\section{${ }^{13} \mathrm{CO}$ observations of candidate massive YSOs in the southern Galactic plane ${ }^{\star}$}

\author{
J. S. Urquhart ${ }^{1}$, A. L. Busfield ${ }^{1}$, M. G. Hoare ${ }^{1}$, S. L. Lumsden $^{1}$, R. D. Oudmaijer ${ }^{1}$, T. J. T. Moore ${ }^{2}$, A. G. Gibb ${ }^{3}$, \\ C. R. Purcell ${ }^{4,5}$, M. G. Burton ${ }^{4}$, and L. J. L. Marechal ${ }^{6}$ \\ 1 School of Physics and Astrophysics, University of Leeds, Leeds LS2 9JT, UK \\ e-mail: jsu@ast. leeds.ac.uk \\ 2 Astrophysics Research Institute, Liverpool John Moores University, Twelve Quays House, Egerton Wharf, \\ Birkenhead CH41 1LD, UK \\ 3 Department of Physics and Astronomy, University of British Columbia, 6224 Aricultural Road, Vancouver, BC, V6T 1Z1, Canada \\ 4 School of Physics and Astronomy, University of New South Wales, Sydney, NSW 2052, Australia \\ 5 Jodrell Bank Observatory, University of Manchester, Cheshire SK11 9DL, UK \\ 6 École Normale Supérieure, Département de Physique, 24 rue Lhomond, 75005 Paris, France
}

Received 6 June 2007 / Accepted 18 August 2007

\section{ABSTRACT}

\begin{abstract}
Context. The Red MSX Source (RMS) survey is an ongoing multi-wavelength observational programme designed to return a large, well-selected sample of massive young stellar objects (MYSOs). We have identified 2000 MYSOs candidates located within our Galaxy by comparing the colours of MSX and 2MASS point sources to those of known MYSOs. The aim of our follow-up observations is to identify other contaminating objects such as ultra compact (UC) HII regions, evolved stars and planetary nebulae (PNe) and distinguish between genuine MYSOs and nearby low-mass YSOs.

Aims. A critical part of our follow-up programme is to conduct ${ }^{13} \mathrm{CO}$ molecular line observations in order to determine kinematic distances to all of our MYSO candidates. These distances will be used in combination with far-IR and (sub)millimetre fluxes to determine bolometric luminosities which will allow us to identify and remove nearby low-mass YSOs. In addition these molecular line observations will help in identifying evolved stars which are weak $\mathrm{CO}$ emitters.

Methods. We have used the $22 \mathrm{~m}$ Mopra telescope, the $15 \mathrm{~m}$ JCMT and the $20 \mathrm{~m}$ Onsala telescope to conduct molecular line observations towards 854 MYSOs candidates located in the $3 \mathrm{rd}$ and 4th quadrants. These observations have been made at the $J=1-0$ (Mopra and Onsala) and $J=2-1$ (JCMT) rotational transition frequency of ${ }^{13} \mathrm{CO}$ molecules and have a spatial resolution of $\sim 20^{\prime \prime}-40^{\prime \prime}$, a sensitivity of $T_{\mathrm{A}}^{*} \simeq 0.1 \mathrm{~K}$ and a velocity resolution of $\sim 0.2 \mathrm{~km} \mathrm{~s}^{-1}$.

Results. We detect ${ }^{13} \mathrm{CO}$ emission towards a total of 752 of the 854 RMS sources observed ( $\left.88 \%\right)$. In total 2132 emission components are detected above $3 \sigma$ level (typically $T_{\mathrm{A}}^{*} \geq 0.3 \mathrm{~K}$ ). Multiple emission profiles are observed towards the majority of these sources -461 sources $(\sim 60 \%)$ - with an average of $\sim 4$ molecular clouds detected along the line of sight. These multiple emission features make it difficult to assign a kinematic velocity to many of our sample. We have used archival CS $(J=2-1)$ and maser velocities to resolve the component multiplicity towards 82 sources and have derived a criterion which is used to identify the most likely component for a further 218 multiple component sources. Combined with the single component detections we have obtained unambiguous kinematic velocities towards 591 sources ( $~ 80 \%$ of the detections). The 161 sources for which we have not been able to determine the kinematic velocity will require additional line data. Using the rotation curve of Brand \& Blitz (1993) and their radial velocities we calculate kinematic distances for all components detected.
\end{abstract}

Key words. stars: formation - stars: early-type - stars: pre-main sequence - ISM: clouds - ISM: kinematics and dynamics

\section{Introduction}

\subsection{Background}

Massive stars $\left(>8 M_{\odot},>10^{4} L_{\odot}\right)$ are responsible for some of the most spectacular phenomena in astrophysics. They inject huge amounts of energy into the interstellar medium (ISM) in the form of UV radiation, molecular outflows, powerful stellar winds and eventually supernova explosions. They are also responsible for depositing large amounts of enriched material back into the ISM. Through these processes massive stars can have an enormous impact on their local environment and can influence the evolution

* Full Table 3 and Fig. 11 are only available in electronic form at the CDS via anonymous ftp to

cdsarc.u-strasbg.fr $(130.79 .128 .5)$ or via

http://cdsweb.u-strasbg.fr/cgi-bin/qcat?]/A+A/474/891 of their host galaxies. They are also thought to play an important role in regulating star formation through the propagation of strong shocks into their surrounding environment. These shocks may be responsible for triggering subsequent generations of star formation or disrupting conditions necessary for star formation in nearby clouds (Elmegreen 1998).

Given the profound impact massive stars have, not only on their local environment, but also on a galactic scale, it is vital that we understand the environmental conditions and processes involved in their birth and the earliest stages of their evolution. Their rarity and short evolutionary time scales means that massive stars are generally located much farther away than low-mass star formation sites. Additionally, massive stars are known to form exclusively in clusters making it difficult to identify and attribute derived quantities to individual sources. Furthermore, 
they reach the main sequence whilst still accreting material, and heavily embedded within their natal molecular cloud, hidden away beneath many magnitudes of visual extinction. For these reasons, until relatively recently, the only catalogue of massive young stellar objects (MYSOs) had been limited to 30 or so serendipitously detected sources (Henning et al. 1984) which are mostly nearby. These difficulties have resulted in our understanding of the earliest stages being far less developed than in the case of low mass star formation, which in broad terms is well understood (Shu et al. 1987, 1993).

The situation has improved considerably in recent years with a number of studies using a variety of selection criteria (e.g., Molinari et al. 1996; Walsh et al. 1997; Sridharan et al. 2002) which have identified many new MYSOs. However, all of these studies are based on IRAS colours which bias them towards bright, isolated sources and tend to avoid dense clustered environments and the Galactic mid-plane where the majority of MYSOs are expected to be found - the scale height of massive stars is $\sim 30^{\prime}$ (Reed 2000). The small numbers of MYSOs identified by these studies, and the way they have been selected, means that they are probably not representative of the general MYSO population and make statistical studies of many aspects of massive star formation difficult. Therefore there is an obvious need for a large, well-selected sample with sufficient numbers of sources in each luminosity bin to allow statistical studies as a function of luminosity. The Red MSX Source (RMS) survey is an ongoing observational programme designed to return just such a sample with complementary data with which to address these issues.

\subsection{The RMS survey}

MYSOs are mid- and far-infrared bright with luminosities of $10^{4}-10^{5} L_{\odot}$ (Wynn-Williams 1982 ). They are likely to be somewhat older than the hot molecular core stage and nuclear fusion has probably begun, however ongoing accretion in some way prevents the ionisation of the surroundings to form an HII region (see Hoare \& Franco 2007). They are almost always associated with massive bipolar molecular outflows (e.g., Wu et al. 2004), another indication that major accretion is likely to be ongoing. MYSOs are also known to possess ionised stellar winds that are weak thermal radio sources $(\sim 1 \mathrm{mJy}$ at $1 \mathrm{kpc}$; Hoare 2002). These objects can therefore be roughly parameterized as near and/or mid-infrared bright, have luminosities consistent with young $\mathrm{O}$ and early B-type stars and are relatively radio continuum quiet with some exceptions (e.g., S:106IR; Drew et al. 1993).

Lumsden et al. (2002) compared the colours of sources from the MSX and 2MASS point source catalogues to those of known MYSOs to develop a colour selection criteria from which we identified approximately 2000 MYSO candidates spread throughout the Galaxy $\left(|b|<5^{\circ}\right)$. Sources toward the Galactic centre were excluded $\left(|l|<10^{\circ}\right)$ due to confusion and difficulties in calculating kinematic distances. A problem with a colour selected sample is that the shape of the spectral energy distribution from an optically thick cloud is insensitive to the type of heating source. Therefore there are several other types of embedded, or dust enshrouded objects, that have similar colours to MYSOs and contaminate our sample, such as ultra compact (UC) HII regions, evolved stars and a small number of planetary nebulae $(\mathrm{PNe})$.

The RMS survey is a multi-wavelength programme of follow-up observations designed to distinguish between genuine MYSOs and these other embedded or dusty objects
(Hoare et al. 2005) and to compile a database of complementary multi-wavelength data with which to study their properties $^{1}$. These include high resolution $\mathrm{cm}$ continuum observations to identify UCHII regions and PNe (Urquhart et al. 2007), mid-infrared imaging to identify genuine point sources, obtain accurate astrometry and avoid excluding MYSOs located near UCHII regions (e.g., Mottram et al. 2007), near-infrared spectroscopy (e.g., Clarke et al. 2006) to distinguish between MYSOs and evolved stars.

Another crucial part of our campaign is to obtain kinematic distances and luminosities towards our whole sample. These allow us to distinguish between nearby low and intermediate mass YSOs from the more massive YSOs. We have therefore conducted a programme of molecular line observations using transitions of the ${ }^{13} \mathrm{CO}$ molecule towards all RMS sources not previously observed or for which good data was not available (see Sect. 2.1 for details). Additionally, these molecular line observations will allow us to eliminate a large proportion of evolved stars since they are not generally associated with strong $\mathrm{CO}$ emission $\left({ }^{12} \mathrm{CO}(J=1-0\right.$ and $J=2-1)$ typically less than $1 \mathrm{~K}$; Loup et al. 1993). In this paper we present the results of our molecular line observations towards 854 RMS sources located in the southern Galactic plane $\left(180^{\circ}<l<350^{\circ}\right)$. In Sect. 2 we outline our source selection, observational and data reduction procedures. We present our results and statistical analysis in Sect. 3. In Sect. 4 we present a summary of the results and highlight our main findings.

\section{Observations and data reduction}

The ${ }^{13} \mathrm{CO}$ transition was chosen for our observations as it is generally found to be only moderately optically thick and is thus better able to probe the bulk parameters of gas along the line of sight than ${ }^{12} \mathrm{CO}$. Moreover, using ${ }^{13} \mathrm{CO}$ avoids many of the problems often encountered when observing with ${ }^{12} \mathrm{CO}$, which is nearly always optically thick and can often result in complex structures with multiple components and/or heavily self-absorbed spectral profiles, particularly towards the Galactic centre. The CS transition was considered as its high critical density (excitation threshold $\sim 10^{4}-10^{5} \mathrm{~cm}^{-3}$ ) makes it an excellent tracer of high density molecular gas. However, CS is much less abundant than ${ }^{13} \mathrm{CO}$ and as a consequence requires significantly longer onsource integration time to obtain an equivalent signal to noise. Additionally, given the lower abundance of CS there is a danger that many of the more distant sources might be missed entirely. Given its higher abundance and low excitation threshold ${ }^{13} \mathrm{CO}$ is generally much stronger than CS and less problematic than ${ }^{12} \mathrm{CO}$ (cf. Jackson et al. 2006; Wu et al. 2001).

\subsection{Source selection}

Of our 2000 colour selected MYSO candidates (hereafter RMS catalogue) 963 are located within the 3rd and 4th quadrants. The large majority of our southern RMS sources were previously unknown in the literature. However, approximately a quarter are associated with bright IRAS point sources, and as such, might have been previously observed as part of another programme. In order to avoid re-observing these sources we conducted an extensive literature search using SIMBAD $^{2}$. This search identified a number of sources for which archival data was already available; the majority of these were found in

\footnotetext{
1 http://ast. leeds.ac.uk/RMS

2 http://simbad.u-strasbg.fr/simbad/sim-fid
} 
two large observational programmes: a CS survey by Bronfman et al. (1996) of colour selected IRAS sources, and a CO survey by Wouterloot \& Brand (1989) which concentrated mainly on IRAS sources located in the outer Galaxy.

Bronfman et al. (1996) conducted a programme of CS $(J=$ 2-1) transition observations towards 1427 IRAS point sources proposed by Wood \& Churchwell (1989) as sites of massive star formation. We have 259 sources in common with sources observed by Bronfman et al. (1996) of which they report 55 as non-detections. The low number of sources in common is because the Wood \& Churchwell (1989) criteria preferentially selects UCHII regions rather than the earlier evolutionary phases of massive star formation. The majority of these were excluded from our observations, but, because CS is generally much harder to detect than ${ }^{13} \mathrm{CO}$ we re-observed all of their non-detections (with the exception of G206.7804-01.9395 for which there was good CO data available). In addition to their non-detections we re-observed $\sim 25 \%$ of their detections to allow for consistency checks and comparison of the two tracers.

Wouterloot \& Brand (1989) used the ${ }^{12} \mathrm{CO}(J=1-0)$ transition to observe 1302 IRAS selected sources located within $10^{\circ}$ of the Galactic plane and between $85^{\circ}<l<280^{\circ}$. We find a total of 76 sources in common between their observations and our southern MYSO candidates. Some of these were excluded but a number were found to possess complex profiles, or were found to be saturated, which led to a large number being re-observed. Using data in the literature we were able to reduce the number of observations needed by over a hundred to 854 .

\subsection{Observation procedures}

We made ${ }^{13} \mathrm{CO}$ observations towards 854 RMS sources from a total of 963 located in the southern Galactic plane. The vast majority of which were observed using the $22 \mathrm{~m}$ Mopra telescope located near Coonabarabran, New South Wales, Australia ${ }^{3}$. The regions of the plane not accessible from Mopra were observed using the $15 \mathrm{~m}$ James Clerk Maxwell Telescope (JCMT) located on Mauna Kea, Hawaii ${ }^{4}$ and the $20 \mathrm{~m}$ Onsala telescope located approximately $50 \mathrm{~km}$ south of Goteborg ${ }^{5}$. The ${ }^{13} \mathrm{CO}(J=$ 1-0) transition was used for the Mopra and Onsala observations, however, the instruments available on the JCMT are unable to operate at this frequency and therefore the higher excitation ${ }^{13} \mathrm{CO}(J=2-1)$ transition was used. The use of these two different transitions should not affect our findings since YSOs are expected to have similar intensities at these frequencies (Little et al. 1994). The number of sources observed by each telescope and the details of each telescopes' observational set-up are summarised in Table 1.

All of these observations were performed in positionswitching mode, with typical on-source integration times of $\sim 10$ min for both the Mopra and Onsala observations, and $\sim 6 \mathrm{~min}$ for the JCMT observations (however, these integration times were reduced for stronger sources). The on-source integration time was split into a number of separate scans consisting

${ }^{3}$ Mopra is operated by the Australia Telescope National Facility, CSIRO and the University of New South Wales during the time of the observations.

${ }^{4}$ The James Clerk Maxwell Telescope is operated by The Joint Astronomy Centre on behalf of the Particle Physics and Astronomy Research Council of the United Kingdom, the Netherlands Organisation for Scientific Research, and the National Research Council of Canada.

${ }^{5}$ Onsala is operated by the Swedish National Facility for Radio Astronomy, Onsala Space Observatory at Chalmers University of Technology.
Table 1. Observational parameters for the ${ }^{13} \mathrm{CO}$ observations.

\begin{tabular}{lccc}
\hline \hline Parameter & Mopra & Onsala & JCMT \\
\hline \# of sources observed & 818 & 9 & 27 \\
Rest frequency $(\mathrm{GHz})$ & 110.201 & 110.201 & 220.3986 \\
Total bandwidth $(\mathrm{MHz})$ & 64 & 80 & 267 \\
Vel. bandpass $\left(\mathrm{km} \mathrm{s}^{-1}\right)$ & 174 & 224 & 360 \\
Number of channels & 1024 & 1600 & 1713 \\
Vel. resolution $\left(\mathrm{km} \mathrm{s}^{-1}\right)$ & 0.17 & 0.14 & 0.21 \\
Beam size (") & 33 & 35 & 21 \\
Date of observations & $2002-2005$ & March 2003 & $2003-2004$ \\
Integration time (mins) & 10 & 10 & 6 \\
Telescope efficiency $\left(\eta_{\mathrm{fss}}\right)$ & 0.55 & 0.43 & 0.69 \\
\hline
\end{tabular}

of $1 \mathrm{~min}$ of on- and off-source integration. Reference positions were offset from source positions by 1 degree in a direction perpendicular to the Galactic plane. These were chosen to avoid contamination of source emission from emission in the reference position at a similar velocity. In some cases, particularly towards the Galactic centre, several positions needed to be tried before a suitable reference position was found.

Mopra was used to observe 818 RMS sources during three periods between 2002 and 2005 . The receiver can be tuned to either single or double side-band mode. The incoming signal is separated into two channels using a polarisation splitter, each of which can be tuned separately allowing two channels to be observed simultaneously. However, the large overheads involved in periodically retuning the second IF between the ${ }^{13} \mathrm{CO}$ and $\mathrm{SiO}$ maser frequency $(86.2 \mathrm{GHz})$, which was used for pointing corrections, meant that for the observations made before 2004 the second IF was set to the maser frequency and was not retuned back to the ${ }^{13} \mathrm{CO}$ frequency between pointing observations. An upgrade of the tuning system in 2004 significantly improved the tuning capabilities of the receiver and made it feasible to use both polarisations when observing our sources which significantly improved the signal-to-noise and lowered integration times.

Of the remaining southern sources, 27 were observed with the JCMT during 2003 and 2004 as part of a long-term bad weather backup programme and nine were observed with the Onsala telescope during a run in March 2003.

Typical system temperatures obtained for both the Mopra and Onsala observations are between 250-450 K depending on weather conditions and telescope elevation, with values typically below $300 \mathrm{~K}$. The higher frequency observations conducted with the JCMT have system temperatures between 400-500 K. Telescope pointing was regularly checked $(\sim 2-3 \mathrm{~h})$ and found to be better than $3^{\prime \prime}$ for the JCMT and $10^{\prime \prime}$ for the Mopra and Onsala telescopes. To correct the measured antenna temperatures $\left(T_{\mathrm{A}}^{*}\right)$ for atmospheric absorption, ohmic losses and rearward spillover and scattering, a measurement was made of an ambient load (assumed to be at $290 \mathrm{~K}$ ) following the method of Kutner \& Ulich (1981); this was done approximately every two hours.

In the upper panel of Fig. 1 we present a plot of the distribution of molecular material as a function of Galactic longitude and latitude as traced by the Dame et al. (2001) ${ }^{12} \mathrm{CO}$ survey. The coloured crosses indicate the RMS positions observed as part of the ${ }^{13} \mathrm{CO}$ observations presented here; we indicate whether the observation resulted in a non-detection, detection of a single or multiple components by colouring the crosses red, blue and green respectively. The RMS sources towards which ${ }^{13} \mathrm{CO}$ emission has been detected correlate extremely well with the distribution of the ${ }^{12} \mathrm{CO}$. In the lower panel of Fig. 1 we present a plot 


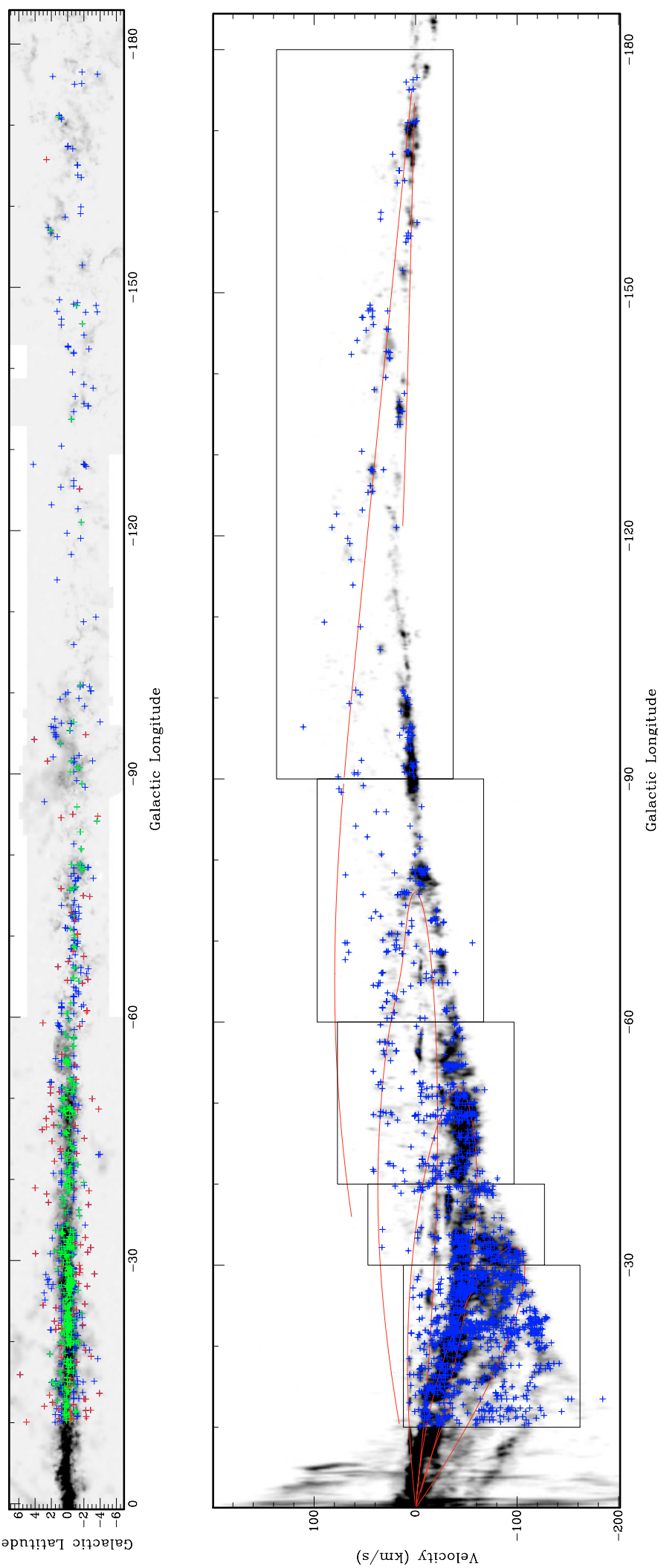

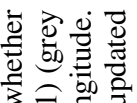

응흐

둥

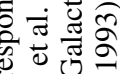

政

政.

石

政

등 의

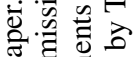

20

记

$\exists \exists$

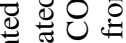

每

og

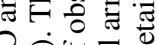

8 केष

$\Xi 50.5$ क

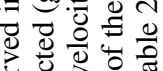

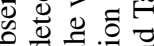

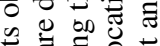

군주

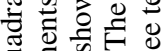

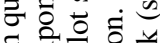

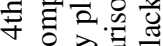

0.5

는응 ․ㅡㅁ

๑芯过

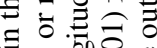

๖

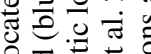

웡

过菏

言苛

क जै छี छ

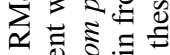

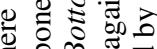

嗼

$0 . \overline{0}$

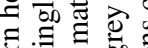

흐. $\cong \cdot$

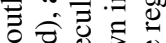

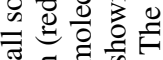

후유

ㅎํ워

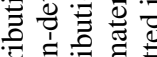

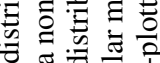

$0 . \Xi \overline{0}$

可造过

ัँญ

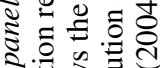

2. 馬方

仓

블 
Table 2. Systemic spectrometer velocities used at different Galactic longitudes to optimise available velocity bandpass. These were chosen from visual inspection of the longitude-velocity maps of Dame et al. (2001).

\begin{tabular}{lc}
\hline \hline Gal. longitude & Centred velocity $\left(\mathrm{km} \mathrm{s}^{-1}\right)$ \\
\hline $180-270$ & 50 \\
$271-300$ & 20 \\
$301-320$ & -10 \\
$321-330$ & -40 \\
$331-350$ & -75 \\
\hline
\end{tabular}

of the distribution of ${ }^{12} \mathrm{CO}$ as a function of Galactic longitude and $V_{\mathrm{LSR}}$ (again taken from Dame et al. 2001). We have overplotted the velocities of all detected components. Additionally, we have overlaid the longitude-velocity of the updated spiral arm model of Taylor \& Cordes (1993) by Cordes (2004) in red to illustrate how their velocities vary as a function of longitude in our Galaxy.

The velocities over which molecular clouds can be found in the southern Galactic plane range from approximately $-150 \mathrm{~km} \mathrm{~s}^{-1}$ to $+100 \mathrm{~km} \mathrm{~s}^{-1}$. Since the total Galactic velocity range $\left(\sim 250 \mathrm{~km} \mathrm{~s}^{-1}\right)$ is larger than the total velocity bandpass available to our Mopra and Onsala observations it was necessary to change the central velocity as a function of Galactic longitude in order to optimise our velocity coverage. We used the Galactic longitude-velocity distribution maps of Dame et al. (2001) to centre the available velocity bandpass as a function of longitude to cover as much of the Galaxy as possible. The central velocities used at various Galactic longitudes are tabulated in Table 2 and the region covered by each are outlined in black on the lower panel of Fig. 1. Comparing the regions covered by our observations with that of the distribution of ${ }^{12} \mathrm{CO}$ shows that, although our velocity bandpass is limited, we have obtained an almost complete coverage of the southern part of the Galaxy.

\subsection{Data reduction}

The basic spectral line data reduction and processing was performed using standard procedures and the software package specific to the individual telescopes used: Dfm (Data From Mopra) ${ }^{6}$ for Mopra data, the $\mathrm{Xs}^{7}$ package for Onsala data and class (Continuum and Line Analysis Single-dish Software) ${ }^{8}$ for JCMT data. As previously mentioned the observation of each source results in between six and ten separate integrations. Sky emission was subtracted from these individual scans which were inspected to remove poor data. The remaining scans were averaged together to produce a single spectrum for each source and a loworder polynomial was subtracted from the baseline. The resulting $1 \sigma$ sensitivity for each spectrum was typically $T_{\mathrm{A}}^{*} \simeq 0.1 \mathrm{~K}$ per channel (see Fig. 2).

The spectral line parameters were obtained by fitting Gaussian profiles to each component present in a given spectrum using Xs. Where necessary, higher-order polynomials were fitted and subtracted from the baselines before the Gaussians were fitted. In the majority of cases a Gaussian profile provided a good fit to the data, however, in a significant number of cases, line components displayed significant deviation from a Gaussian

${ }^{6}$ A tcl/tk graphical interface written for Spc (Spectral Line Reduction Package) by C. Purcell.

7 Written by P. Bergman, Onsala Space Observatory.

${ }^{8}$ Part of the Gildas (Grenoble Image and Line Data Analysis Software) working group software.

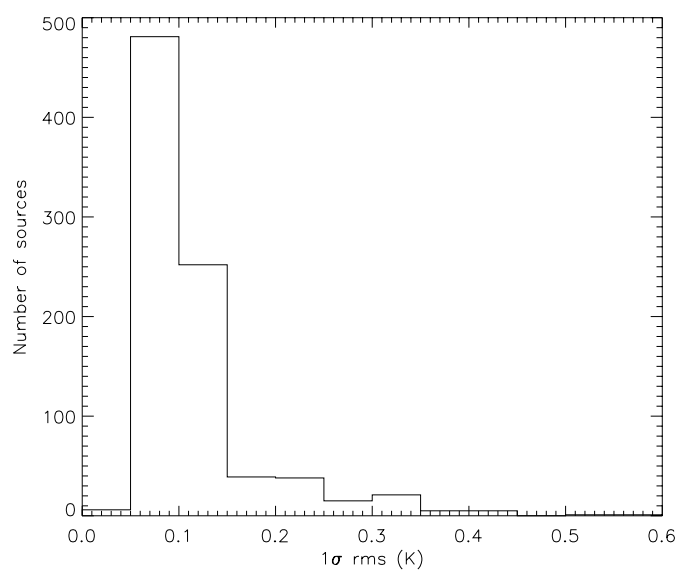

Fig. 2. Histograms of $1 \sigma \mathrm{rms}$ obtained from 6-10 min observations using a bin size of $0.05 \mathrm{~K}$. Over $90 \%$ of the observations have a sensitivity of better than $0.15 \mathrm{~K}$ with typical values of $\sim 0.1 \mathrm{~K}$.

profile to warrant an additional comment. We have adopted the classification scheme used by Wouterloot \& Brand (1989) for their outer Galaxy ${ }^{12} \mathrm{CO}$ observations of colour selected IRAS sources. They distinguish seven different non-Gaussian profiles which are: flat top, asymmetric blue or red top, blue or red asymmetry, self-absorption, blue and/or red wing, blue or red shoulder, self-absorption. However, assigning these can be a very subjective exercise and care must be taken when drawing conclusions from any analysis performed. For example, in some cases the distinction between some of the profiles can be quite arbitrary such as between asymmetric top and shoulder profiles. Moreover, some emission features can exhibit several different kinds of profile simultaneously. In Fig. 3 we present some examples of the emission profiles observed towards RMS sources.

In addition to the profiles just mentioned we encountered a number of cases where two or more separate profiles in close proximity overlap with each other to form complex features, or where the shoulder component was particularly pronounced and a single Gaussian produced a poor fit to the data. In these cases it is not clear whether these represent the superposition of a number of clouds along the line of sight or supersonic motions within a particular cloud. However, their close proximity in velocity and resulting kinematic distances (see Sect. 3.3) would suggest that if they are not associated with the same cloud, they are probably part of the same molecular complex. In some cases it is difficult, if not impossible, to distinguish between a profile that appears to be self-absorbed and two separate, but overlapping components. We identify sources as blended features if either source overlaps with the other above the half-power level.

Finally the data were calibrated to the corrected receiver temperature scale $T_{\mathrm{R}}^{*}$, which accounts for loses due to forward spillover and scattering, by dividing the antenna temperatures by the telescope efficiency where $T_{\mathrm{R}}^{*}=T_{\mathrm{A}}^{*} / \eta_{\mathrm{fss}}$, and $\eta_{\mathrm{fss}}$ is the forward spillover and scattering efficiency (see Table 1 for individual telescope efficiencies). Since the main objective of these observations is to obtain kinematic velocities, rather than accurate line intensities, little absolute calibration was performed. We therefore estimate the calibration uncertainties could be as much as a factor of two.

In a small number of cases some of our spectra have been contaminated by emission present in the reference position which was not evident in the individual integrations and only became apparent when they were averaged together. The data obtained for two sources (i.e., G336.0778+00.0361 and 

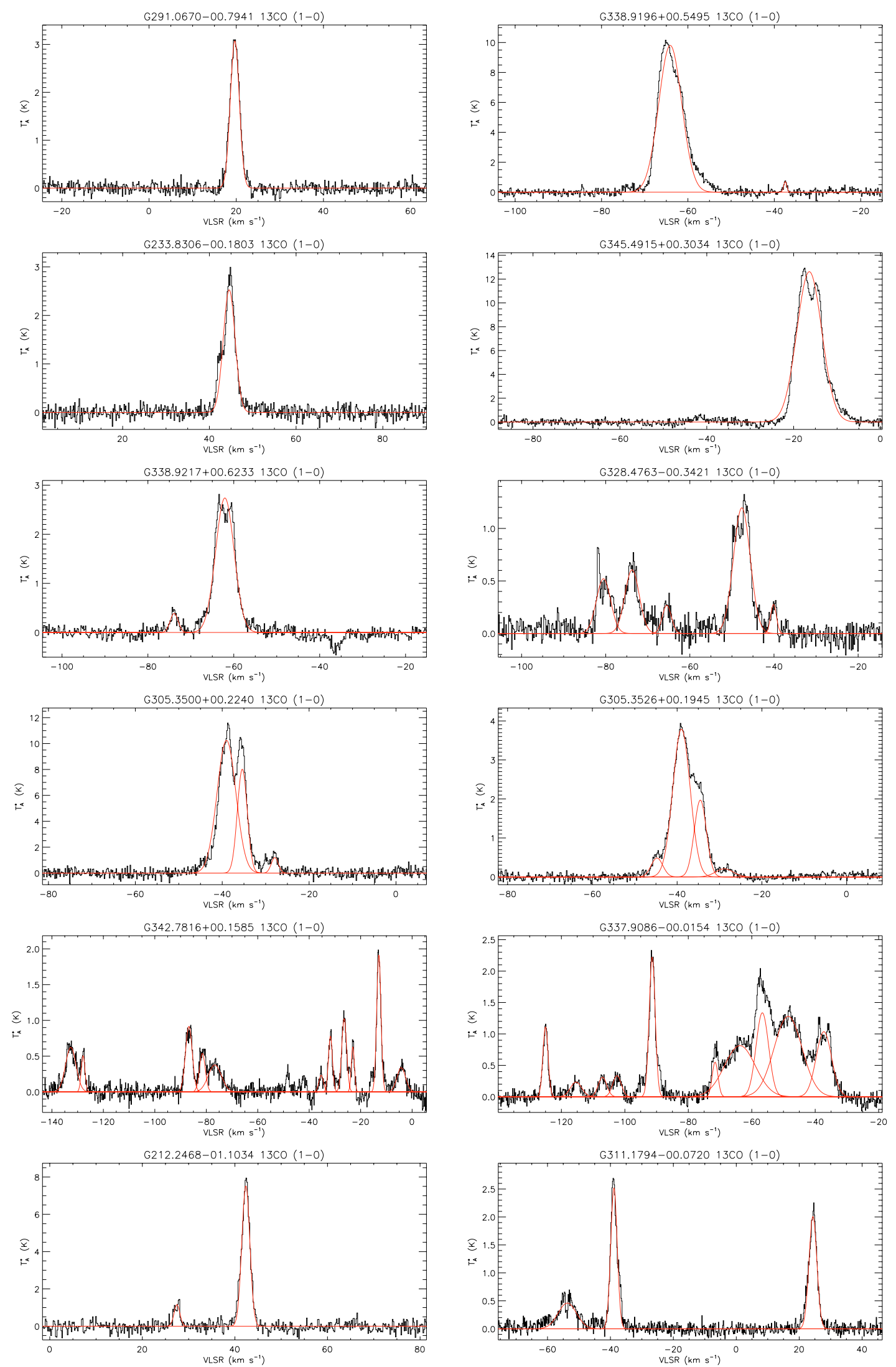

Fig. 3. Example spectra observed towards RMS sources. The spectra presented in the top four rows have been chosen to illustrate the different kinds of emission profiles exhibited in the data and to clarify the classification scheme used for the non-Gaussian profiles. Moving left to right from the top left the profiles are classified as follows: Gaussian, red wing, blue shoulder, self-absorbed, flat-top, red asymmetic flat-top, blended and blended feature with a main and shoulder component. The bottom two rows show examples of multiple components seen towards the majority of our sample. 


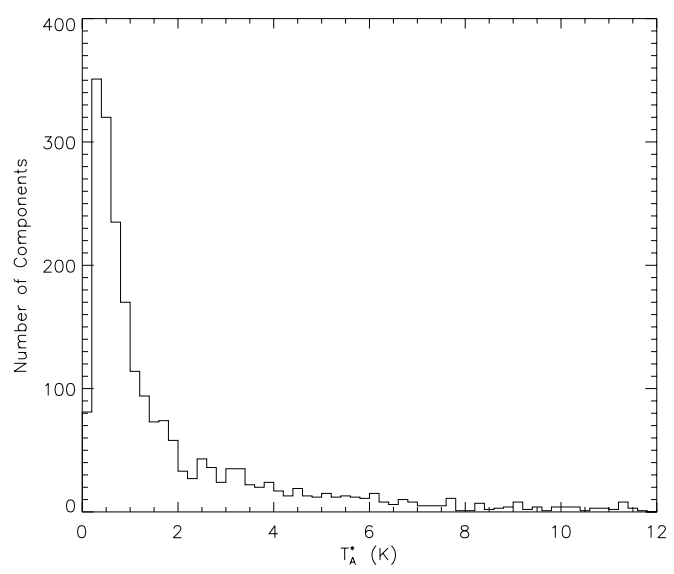

Fig. 4. Histogram of the distribution of $T_{\mathrm{A}}^{*}$ measured towards RMS sources. The hard cutoff under $0.25 \mathrm{~K}$ on the temperature plot is due to the sensitivity limit. The bin size is $0.2 \mathrm{~K}$.

G338.4921+00.0683) were corrupted and will not be presented here.

\section{Results and analysis}

\subsection{Detection statistics and distributions}

We detected ${ }^{13} \mathrm{CO}$ emission towards 752 of the 854 RMS sources observed ( $\sim 88 \%)$ with just over a 100 non-detections. Multiple emission profiles are observed towards the majority of these sources - 461 sources $(\sim 60 \%)$ - with an average of $\sim 4$ components found in each spectrum. A total of 2132 emission components are detected above $3 \sigma$ (typically $T_{\mathrm{A}}^{*} \geq 0.3 \mathrm{~K}$ ). In Figs. 4 and 5 we present histograms of the peak temperatures $\left(T_{\mathrm{A}}^{*}\right)$ and $F W H M$ of all detected components respectively. The hard cutoff at low $T_{\mathrm{A}}^{*}$ seen in Fig. 4 is due to the observational detection limit. The average temperature is $\sim 2 \mathrm{~K}$ and $F W H M$ is $\sim 4 \mathrm{~km} \mathrm{~s}^{-1}$, however, both distributions are highly skewed to large values and consequently more typical (i.e., median) values are $\sim 0.7 \mathrm{~K}$ and $2.5 \mathrm{~km} \mathrm{~s}^{-1}$ respectively. In Fig. 6 we present the distribution of integrated intensities $\left(\int T_{\mathrm{R}} \mathrm{d} V=1.06 T_{\mathrm{R}}^{*} \mathrm{~d} v\right.$, where $\mathrm{d} v$ is the $F W H M$ of the line) of all detected components.

We present the parameters obtained from Gaussian fits to the data and derived values in Table 3 and present plots of the fitted spectra in Fig. 11 (only available in elecronic form at the CDS). The table format is as follows: the RMS names, positions and observations $1 \sigma$ rms in Cols. 1-4; component number and parameters extracted from Gaussian fits (i.e., $V_{\mathrm{LSR}}, T_{\mathrm{R}}^{*}$ and $F W H M$ ) and profile type in Cols. 5-9; component integrated intensity in Col. 10; in Cols. 11-13 we present the Galactic centre radius and kinematic distances determined from the Brand \& Blitz (1993) rotation model (see Sect. 3.3 for details).

The spatial distributions of the non-detections, single and multiple component detections can be seen in the Galactic longitude-latitude plot presented in the upper panel of Fig. 1. In this plot the multiple component detections are clearly concentrated not only towards the Galactic centre but also have a very narrow latitude range. The distribution of the single peak detections are more evenly spread in Galactic longitude but still show a narrow latitude range. The non-detections appear to be evenly distibuted in both longitude and latitude. The different latitude distributions can be clearly seen in the histograms presented in Fig. 7. The distribution of the multiple component sources, which constitute the majority of the detections, is

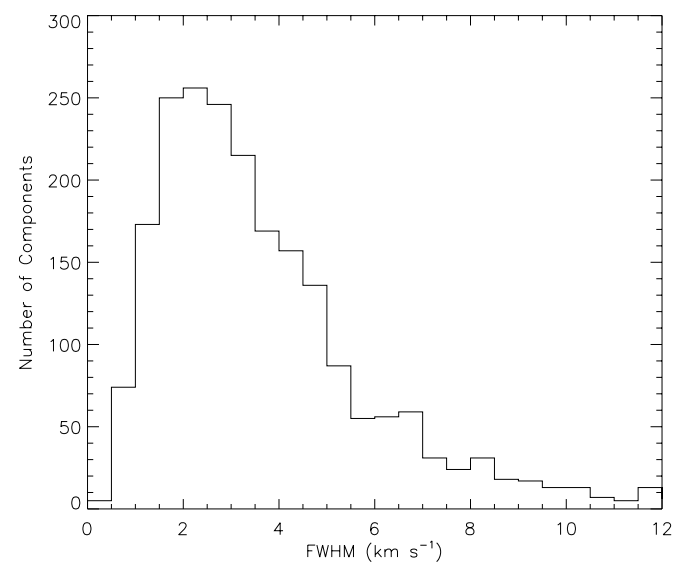

Fig. 5. Histogram showing the distribution of $F W H M$ observed towards RMS sources. The distribution peaks at $\sim 2.5 \mathrm{~km} \mathrm{~s}^{-1}$. Bin size used is $0.5 \mathrm{~km} \mathrm{~s}^{-1}$.

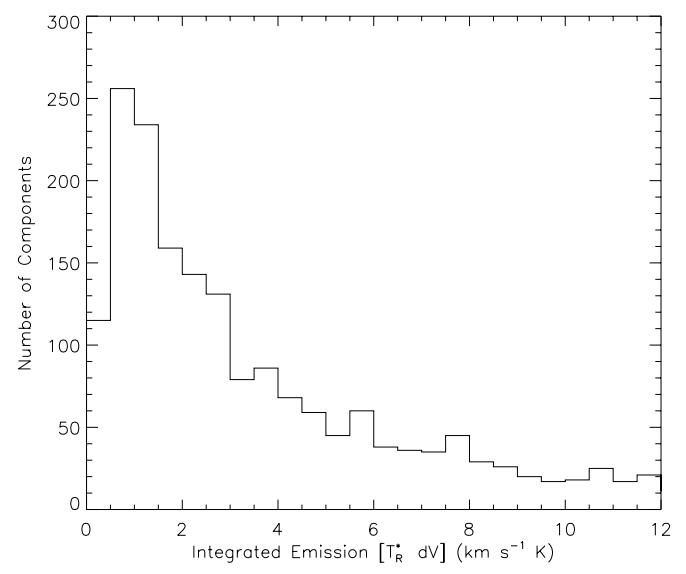

Fig. 6. Histogram of integrated intensity $\left(\int T_{\mathrm{R}}^{*} \mathrm{~d} V\right)$ of all components detected towards RMS sources.

strongly peaked around the Galactic mid-plane with nearly all of these sources confined within two degrees of the plane, and the majority within one degree. The single component detections are also strongly peaked, but have a much broader latitude distribution. The non-detections appears to be evenly distributed in latitude; these sources are most likely evolved stars which have similar colours to embedded YSOs but have very little, if any, associated molecular material. On closer examination of the distribution of non-detections there appears to be a minimum at $|b| \simeq 0^{\circ}$ possibly indicating an unfortunate line of sight alignment between a small number of evolved stars and molecular clouds towards the Galactic mid-plane. However, these will be identified through our near infrared spectroscopic observations.

\subsection{Identification of multiple components}

Although using the ${ }^{13} \mathrm{CO}$ transition has proved successful in detecting emission towards potential MYSOs one significant drawback is the large number of components detected towards the majority of our sample. A histogram of the number of components detected towards each of RMS source is presented in Fig. 8. As previously mentioned we re-observed a number of sources towards which Bronfman et al. (1996) has reported detecting CS emission. In sources towards which only a single CS and ${ }^{13} \mathrm{CO}$ component have been detected we find their velocities agree to better than $1 \mathrm{~km} \mathrm{~s}^{-1}$. Using this velocity correlation we 


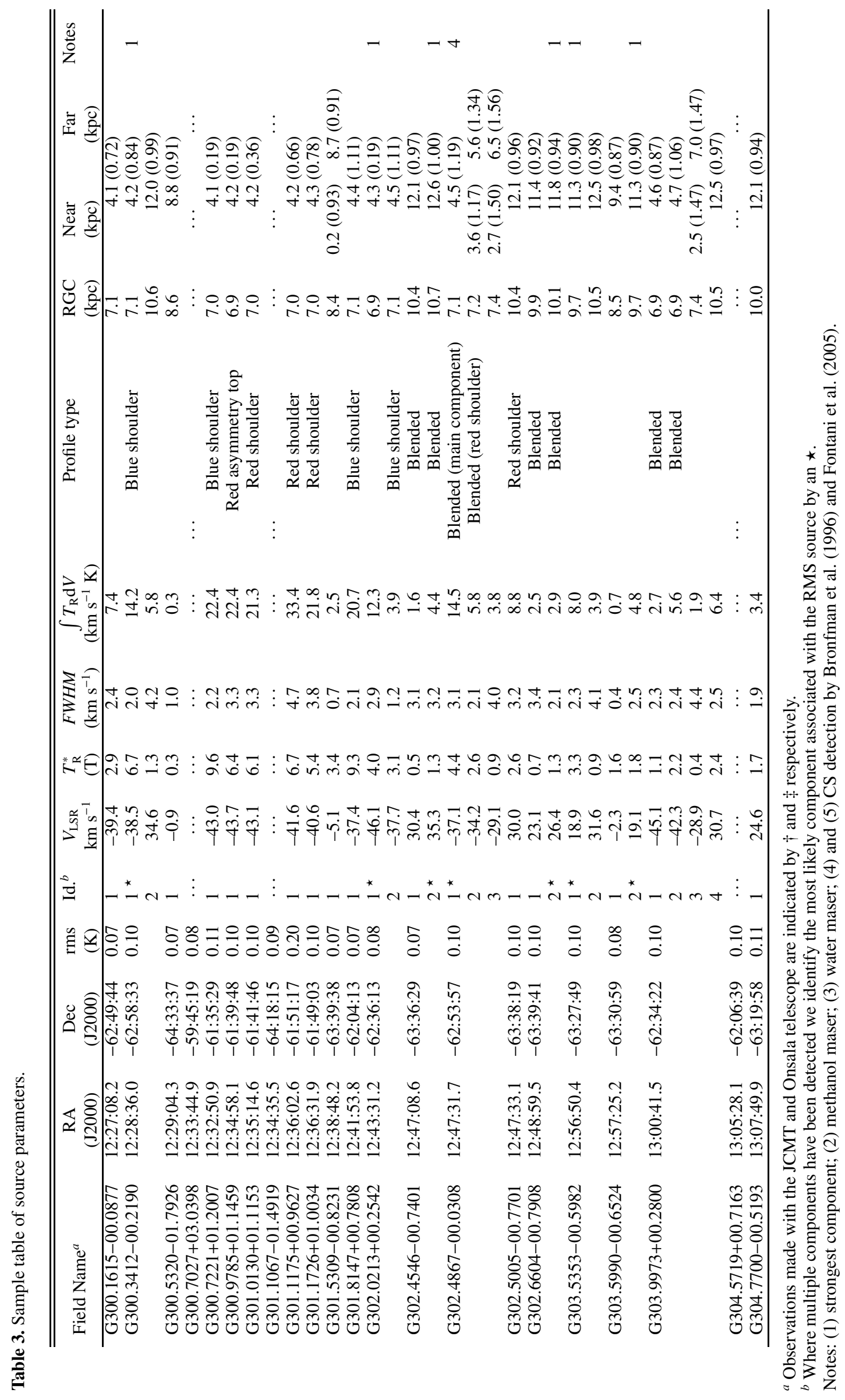



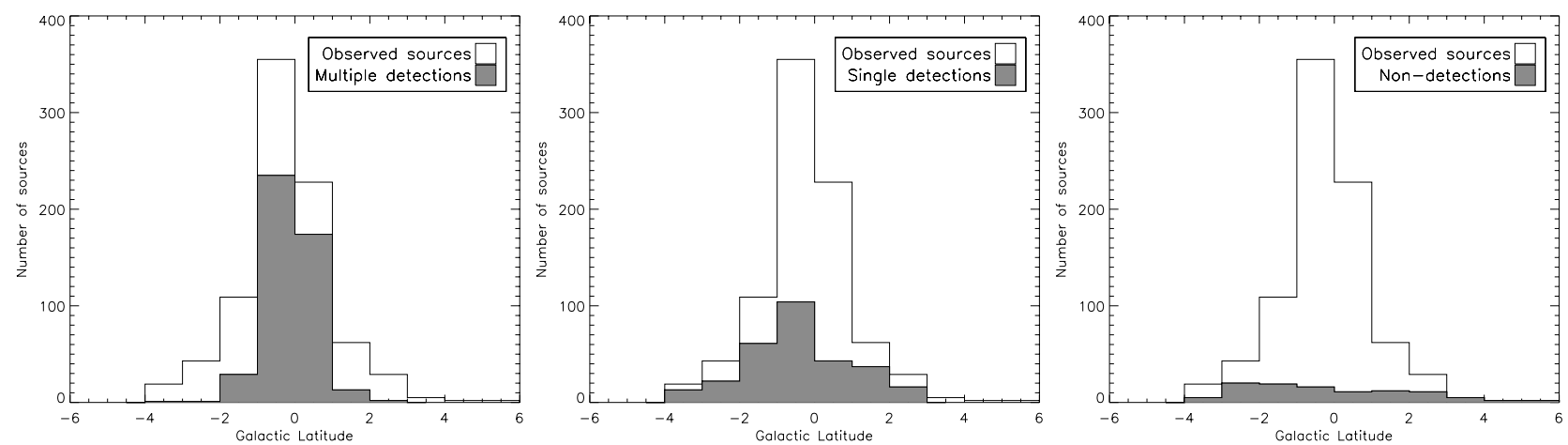

Fig. 7. Histograms of the Galactic latitude distribution of observed RMS sources. The unfilled histogram in each plot consists of all sources observed and reported in this paper. Histograms of the latitude distributions of the multiple component detections, single component detections and non-detections are over-plotted (grey histogram) on the left, middle and right panels respectively. A bin size of 1 degree has been used for all of these histograms.

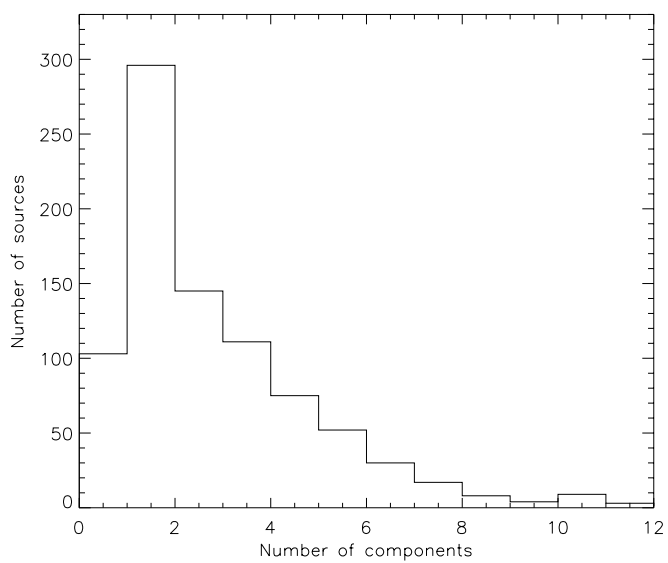

Fig. 8. Histogram of the number of components detected towards RMS sources reported in this paper.

are able to identify the most likely component associated with an RMS source where multiple components are detected in 18 cases using CS $(J=2-1)$ results found in the literature (i.e., Bronfman et al. 1996; Fontani et al. 2005). Additionally, in a number of cases the associated component can easily be identified as they are far stronger than any other component seen in the spectrum. However, identifying the correct component is not always so obvious, and before we can assign kinematic distances to our MYSOs candidates we need to find a way to resolve these multi-component sources. In the bottom panels of Fig. 3 we present two examples of multiple component detections. In the left hand panel it is easy to identify the component associated with the RMS source, however, in the panel on the right two of the components are approximately equal in strength making it difficult to identify the one associated with the RMS source.

Water and methanol masers are often associated with massive star forming regions and their velocities may help identify the component of interest towards some of these multiple component detections. Maser emission can consist of many components spread over a wide range of velocities, typically $10-15 \mathrm{~km} \mathrm{~s}^{-1}$ for methanol and up to $70 \mathrm{~km} \mathrm{~s}^{-1}$ for water masers (Sridharan et al. 2002). Despite these large velocity ranges and number of components, the velocity of the brightest component correlates extremely well with the velocity of the molecular clouds with which they are associated. In order to derive a velocity criterion with which to associate masers with a particular molecular component we cross-matched methanol masers from the Pestalozzi et al. (2005) catalogue and water masers from the Arcetri catalogue (Valdettaro et al. 2001) with CS detections reported by Bronfman et al. (1996). In order to see how the velocities of the molecular clouds and masers varied as a function of angular separation between them we used three search radii $\left(120^{\prime \prime}, 60^{\prime \prime}\right.$ and $\left.15^{\prime \prime}\right)$ to match masers and CS sources. We found 233 methanol masers and 148 water masers in common with the CS sources within the $120^{\prime \prime}$ search radius. In Fig. 9 we present histograms of the differences in the peak methanol and water maser velocities compared to the CS velocities as a function of angular separation.

These histograms reveal a fairly robust correlation, even at rather large angular separations, between the observed velocities of CS emission, which gives the velocity of the molecular cloud, and the velocities of nearby masers. These plots show that both distributions level off at approximately the same $\Delta V\left(\sim 15 \mathrm{~km} \mathrm{~s}^{-1}\right)$ with standard deviations of $\sim 2.5 \mathrm{~km} \mathrm{~s}^{-1}$ and $\sim 3.5 \mathrm{~km} \mathrm{~s}^{-1}$ for methanol and water masers respectively for all of the angular distributions plotted (source with velocity differences greater than $200 \mathrm{~km} \mathrm{~s}^{-1}$ have been excluded). It is therefore possible to identify the most likely $\mathrm{CO}$ component in cases where the difference in velocity between any two $\mathrm{CO}$ components is large and the velocity difference between a particular $\mathrm{CO}$ component and the maser velocities is smaller than three times the standard deviation $\left(3 \sigma \simeq 7.5 \mathrm{~km} \mathrm{~s}^{-1}\right.$ and $10.5 \mathrm{~km} \mathrm{~s}^{-1}$ for methanol and water masers respectively).

We have used these $3 \sigma$ values to identify the most likely star forming cloud where multiple components have been detected and either a methanol or water maser has been found within $120^{\prime \prime}$. We found 78 methanol masers and 12 water masers associated with 79 RMS sources and are able use this association criterion to resolve the multiple component ambiguity towards 64 sources. Using a combination of the archival maser and CS data we are able to identify the correct component associated with an RMS source in 82 of the 461 multiple component cases. Although using these other tracers of massive star formation has proved useful in reducing the number of multiple component sources by $\sim 20 \%$, the vast majority remain unresolved. We notice that in cases where we have been able to associate a component with an RMS source it is nearly always the component with the largest integrated intensity $\left(\int T_{\mathrm{R}} \mathrm{d} V\right)$ that is identified by the maser or CS velocity. In fact the masers and CS lines are almost exclusively associated with the strongest components (in 78 of the 82 cases solved) and only in a few cases (4) is the emission associated with slightly weaker components, but never less than half that of the strongest component. Therefore we can 

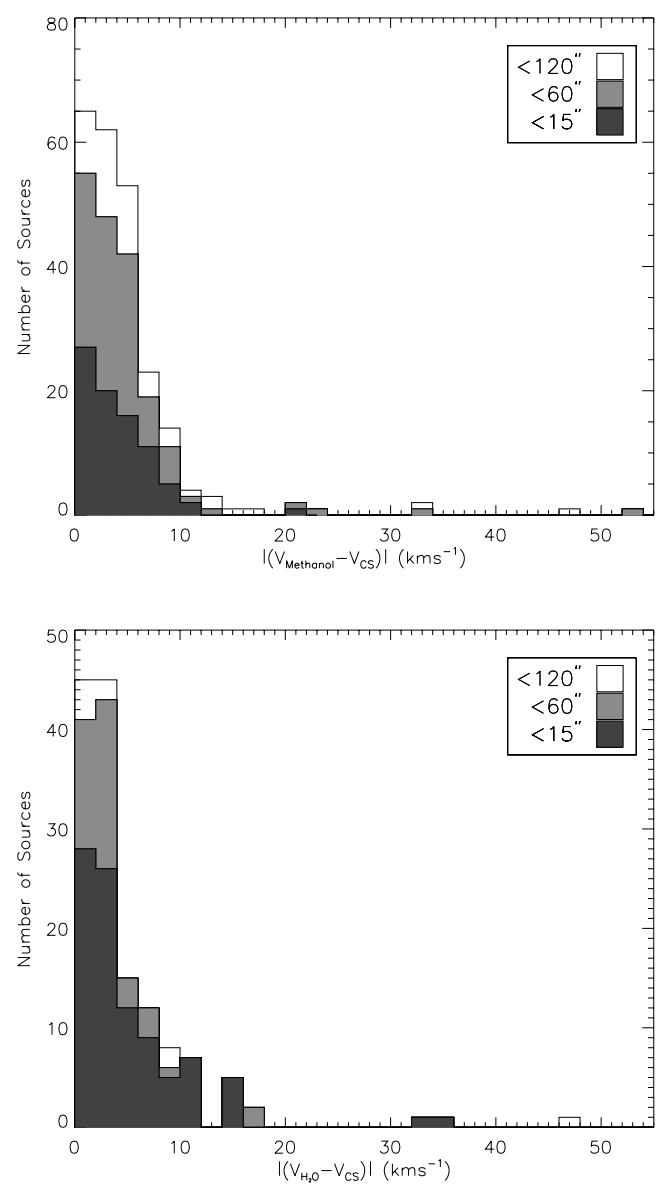

Fig. 9. Histogram illustrating the difference between the velocities of methanol (upper panel) and water masers (lower panel) and the velocities of their host molecular clouds as traced by CS (see text for details). The bin size is $2 \mathrm{~km} \mathrm{~s}^{-1}$ and the total number of sources used in each plot are 233 and 148 for methanol and water masers respectively. The unfilled histogram includes all maser-CS matches within a $120^{\prime \prime}$ search radius, over-plotted in light and dark grey include all matches with in a $60^{\prime \prime}$ and $15^{\prime \prime}$ radius respectively. The velocity range of the water-CS sources have been truncated to facilitate comparison between the to distributions shown, however, only a few sources have velocity differences of greater than the range plotted.

use this information to identify the most likely component associated with the MYSO by picking the strongest component as long as it is more than twice as strong as any other component present in the data.

By applying this criterion to our data we are able to resolve the component ambiguity towards a further 218 sources, bringing the total number resolved to 300 or $65 \%$ of the multicomponent detections. Together with the single component detections we have been able to determine kinematic velocities towards $\sim 80 \%$ of our MYSO candidates. For sources where we are able to resolve the component multiplicity we indicate the most likely component associated with an asterisk in the component number column of Table 3. Additional observational data will be required to resolve the remaining 161 sources towards which multi-components are detected. A number of these may be resolved using data from the Methanol Multibeam (MMB) survey $)^{9}$ which is currently underway and plans to survey the whole Galactic plane for $|b|<2^{\circ}$. For the remaining sources we

\footnotetext{
${ }^{9}$ http://www.jb.man.ac.uk/research/methanol
}

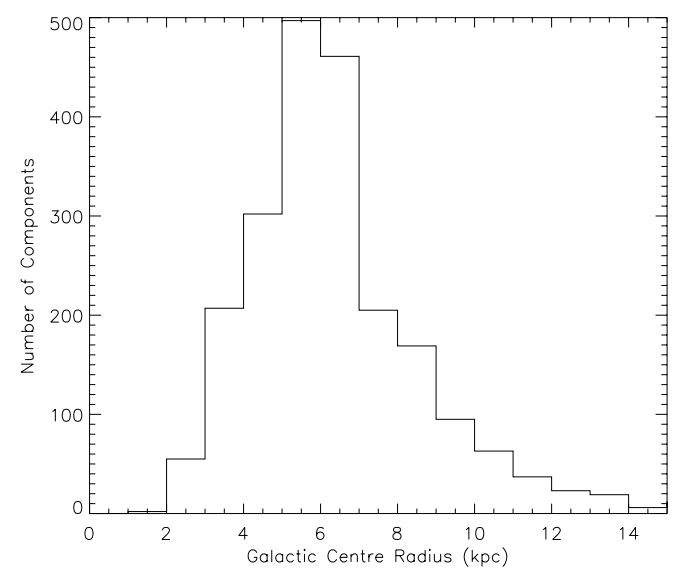

Fig. 10. Histogram showing the distribution of clouds with respected to their distance from the Galactic centre. The distribution peaks between 5 and $6 \mathrm{kpc}$ consistent with the location of the $5 \mathrm{kpc}$ molecular ring which surrounds the Galactic centre. The bin size used is $1 \mathrm{kpc}$.

plan to resolve their component ambiguities with a programme of CS, water masers and CO mapping observations.

\subsection{Kinematic distances}

We have used the rotation curve of Brand \& Blitz (1993) to calculate kinematic distances and Galactocentric radii to all detected components. We have assumed the distance to the Galactic centre is $8.5 \mathrm{kpc}$ and a solar velocity of $220 \mathrm{~km} \mathrm{~s}^{-1}$. In Fig. 10 we present a histogram of the Galactic radii of all detected components, the distribution peaks between 5 and $6 \mathrm{kpc}$, consistent with the location of the thick ring of material which surrounds the centre of the Galaxy. The vast majority of sources in our catalogue are located within the solar circle $(\sim 80 \%)$ and therefore their $V_{\mathrm{LSR}}$ results in two possible distances equally spaced on either side of the tangent point; these are referred to as the near and far distances. This is known as the kinematic distance ambiguity problem and makes determining accurate distances to objects located within the solar circle difficult. The only sources within the solar circle that do not suffer from this ambiguity are sources located at the tangent point.

The Galactocentric radii and kinematic distances determined from the rotation model for all sources located between $192^{\circ}<$ $l<350^{\circ}$ are presented in Cols. $11-13$ of Table 3 . We have not presented distances for sources located near the Galactic anticentre since they are very uncertain. Sources with components outside the solar circle have only a single distance as do sources located at the tangent points. Errors in the distances have been determined by shifting the component $V_{\mathrm{LSR}}$ by $\pm 10 \mathrm{~km} \mathrm{~s}^{-1}$ to account for peculiar motions; these result in typical errors of $\sim 1 \mathrm{kpc}$. If the near and far distances for a particular component are within $1 \mathrm{kpc}$ of each other we place it at the tangent point. In total 97 components were found to be located at or near the tangent point leaving 1679 components with ambiguous distances.

There are a number of methods discussed in the literature that can be applied to solve the distance ambiguity such as: $\mathrm{H}_{2} \mathrm{CO}$ (formaldehyde) absorption (e.g., Downes et al. 1980; Araya et al. 2001, 2002) or radio recombination lines in conjunction with HI absorption toward HII regions (e.g., Kolpak et al. 2003), and HI self-absorption and molecular line emission towards molecular clouds and MYSOs (e.g., Jackson et al. 2002; Busfield et al. 2006). The HI self-absorption method has already been successfully used to resolve distance ambiguities towards a 
subset of the RMS sample (see Busfield et al. 2006 for details). We will use the HI methods to resolve the kinematic distance ambiguity towards the remaining sources in a subsequent paper.

\section{Summary}

We present the results of a programme of molecular line observations of towards 854 candidate massive young stellar objects (MYSOs) located in the 3rd and 4th quadrant of the Galaxy. These have been made using the Mopra and Onsala telescopes and the JCMT using the $J=1-0$ and $J=2-1$ rotational transitions of the ${ }^{13} \mathrm{CO}$ molecules. We detect emission towards 752 MYSOs with multiple components being seen in $60 \%$ of cases. The multiple detections are concentrated within $2^{\circ}$ of the Galactic mid-plane and strongly peaked within $1^{\circ}$. The single component detections are also strongly peaked but have a slightly broader distribution in Galactic latitude $|b| \pm 3^{\circ}$. The non-detections are evenly distributed over a latitude range of $\pm 4^{\circ}$, consistent with them being identified as evolved stars.

We have used archival methanol and water maser, and CS ( $J=2-1)$ molecular line velocities to resolve the component ambiguity towards 82 MYSO candidates where multiple components have been detected. Using the results of our maser and CS associations we have derived a criterion for selecting the most likely component for sources where no useful tracer is present. In total we have been able to identify the component associated with our MYSO candidates in 300 cases of the 461 multiple detections. Combined with the single component detections we have obtained unambiguous kinematic velocities towards 591 sources ( $~ 80 \%$ of the detections). The 161 sources for which we have not been able to determine the kinematic velocity will require additional line data.

These observations of southern sources form part of a larger programme of follow-up observations of near and mid-infrared colour selected sample of MYSO candidates. The results of observations made towards sources in the northern Galactic plane will be presented in a subsequent paper.

Acknowledgements. The authors would like to thank the Director and staff of the Paul Wild Observatory, the JCMT and Onsala for their hospitality and assistance during our observing runs. We would also like to thank Thomas Dame for providing the integrated ${ }^{12} \mathrm{CO}$ maps used in Fig. 1. J.S.U. and C.R.P. are supported by PPARC postdoctoral fellowship grants; C.R.P. was also supported by a UNSW Scholarship during the observing. This research would not have been possible without the SIMBAD astronomical database service operated at CDS, Strasbourg, France and the NASA Astrophysics Data System Bibliographic Services.

\section{References}

Araya, E., Hofner, P., Churchwell, E., \& Kurtz, S. 2001, BAAS, 33, 1487 Araya, E., Hofner, P., Churchwell, E., \& Kurtz, S. 2002, ApJS, 138, 63

Brand, J., \& Blitz, L. 1993, A\&A, 275, 67

Bronfman, L., Nyman, L.-A., \& May, J. 1996, A\&AS, 115, 81

Busfield, A. L., Purcell, C. R., Hoare, M. G., et al. 2006, MNRAS, 366, 1096

Clarke, A. J., Lumsden, S. L., Oudmaijer, R. D., et al. 2006, A\&A, 457, 183

Cordes, J. M. 2004, in Milky Way Surveys: The Structure and Evolution of our

Galaxy, ed. D. Clemens, R. Shah, \& T. Brainerd, ASP Conf. Ser., 317, 211

Dame, T. M., Hartmann, D., \& Thaddeus, P. 2001, ApJ, 547, 792

Downes, D., Wilson, T. L., Bieging, J., \& Wink, J. 1980, A\&AS, 40, 379

Drew, J. E., Bunn, J. C., \& Hoare, M. G. 1993, MNRAS, 265, 12

Elmegreen, B. G. 1998, in Origins, ed. C. E. Woodward, J. M. Shull, \& H. A. Thronson, Jr., ASP Conf. Ser., 148, 150

Fontani, F., Beltrán, M. T., Brand, J., et al. 2005, A\&A, 432, 921

Henning, T., Friedemann, C., Guertler, J., \& Dorschner, J. 1984, Astron. Nachr., 305,67

Hoare, M. G. 2002, in Hot Star Workshop III: The Earliest Phases of Massive Star Birth, ed. P. Crowther, ASP Conf. Ser., 267, 137

Hoare, M. G., \& Franco, J. 2007, Diffuse Matter from Star Forming Regions to Active Galaxies - A Volume Honouring John Dyson, ed. T. W. Hartquist, J. M. Pittard, \& S. A. E. G. Falle, Series: Astrophys. Space Sci. Proc. (Dordrecht: Springer), 61

Hoare, M. G., Lumsden, S. L., Oudmaijer, R. D., et al. 2005, in Massive Star Birth: A Crossroads of Astrophysics, ed. R. Cesaroni, M. Felli, E. Churchwell, \& M. Walmsley, IAU Symp., 227, 370

Jackson, J. M., Bania, T. M., Simon, R., et al. 2002, ApJ, 566, L81

Jackson, J. M., Rathborne, J. M., Shah, R. Y., et al. 2006, ApJS, 163, 145

Kolpak, M. A., Jackson, J. M., Bania, T. M., Clemens, D. P., \& Dickey, J. M. 2003, ApJ, 582, 756

Kutner, M. L., \& Ulich, B. L. 1981, ApJ, 250, 341

Little, L. T., Gibb, A. G., Heaton, B. D., Ellison, B. N., \& Claude, S. M. X. 1994, MNRAS, 271, 649

Loup, C., Forveille, T., Omont, A., \& Paul, J. F. 1993, A\&AS, 99, 291

Lumsden, S. L., Hoare, M. G., Oudmaijer, R. D., \& Richards, D. 2002, MNRAS, 336,621

Molinari, S., Brand, J., Cesaroni, R., \& Palla, F. 1996, A\&A, 308, 573

Mottram, J. C., , Hoare, M. G., Lumsden, S. L., et al. 2007, [arXiv: 0709. 2040]

Pestalozzi, M. R., Minier, V., \& Booth, R. S. 2005, A\&A, 432, 737

Reed, B. C. 2000, AJ, 120, 314

Shu, F., Najita, J., Galli, D., Ostriker, E., \& Lizano, S. 1993, in Protostars and Planets III, ed. E. H. Levy, \& J. I. Lunine, 3

Shu, F. H., Adams, F. C., \& Lizano, S. 1987, ARA\&A, 25, 23

Sridharan, T. K., Beuther, H., Schilke, P., Menten, K. M., \& Wyrowski, F. 2002, ApJ, 566, 931

Taylor, J. H., \& Cordes, J. M. 1993, ApJ, 411, 674

Urquhart, J. S., Busfield, A. L., Hoare, M. G., et al. 2007, A\&A, 461, 11

Valdettaro, R., Palla, F., Brand, J., et al. 2001, A\&A, 368, 845

Walsh, A. J., Hyland, A. R., Robinson, G., \& Burton, M. G. 1997, MNRAS, 291, 261

Wood, D. O. S., \& Churchwell, E. 1989, ApJS, 69, 831

Wouterloot, J. G. A., \& Brand, J. 1989, A\&AS, 80, 149

Wu, Y., Wu, J., \& Wang, J. 2001, A\&A, 380, 665

Wu, Y., Wei, Y., Zhao, M., et al. 2004, A\&A, 426, 503

Wynn-Williams, C. G. 1982, ARA\&A, 20, 587 